\title{
Nouveaux progrès en électrodialyse
}

\author{
par \\ G. GUERIF
}

\section{INTRODUCTION}

Notre Société commercialise depuis 1972 des électrodialyseurs sous la marque déposée Aqualyzer. Une part importante de notre activité concerne la déminéralisation et la déacidification des sousproduits laitiers (lactosérums, eaux-mères de lactoserie, perméats de lait ou de sérum, etc.).

Depuis cette époque, de nombreuses améliorations ont été apportées à nos appareils, toutefois, plus récemment, d'importants progrès technologiques ont vu le jour tant au niveau du module d'électrodialyse qu'à celui du procédé.

\section{AMELIORATION DU MODULE}

\section{a) Au niveau du cadre séparateur}

Un empilement (ou module) d'électrodialyse est constitué par l'assemblage de membranes alternativement cationique et anionique séparées par un " cadre séparateur ».

Ce dernier joue un rôle essentiel dans l'optimisation du procédé.

Nous rappelons que nous exploitons une technologie originale qui consiste à avoir un espace très faible entre chaque membrane (inférieur à $0,4 \mathrm{~mm}$ ).

Nous demandons aux cadres séparateurs d'assurer plusieurs fonctions :

- séparation des membranes de façon à assurer un écoulement en nappe entre celles-ci ; mure :

- irrigation des compartiments alternativement produit-sau-

- étanchéité vers l'extérieur;

Sodeteg, Zone Industrielle Nord, 283, rue de la Minière, B.P. 24 - 78530 Buc. 
- étanchéité interne, c'est-à-dire pas de communication entre le produit traité et la saumure.

C'est sur ce dernier point que nous avons porté notre effort. En effet, si pour certaines applications comme le dessalement des eaux saumâtres, on peut s'accommoder d'un défaut d'étanchéité interne, dans le cas des lactosérums, il est important de ne pas perdre de matières nobles (lactose, protéines), et de ne pas diluer le produit traité.

Une étude systématique a permis de mettre en évidence la zone du cadre séparateur responsable de la fuite interne. Cette zone est située au niveau des diffuseurs et correspond à une sorte de discontinuité dans l'empilement des plans de point (voir fig. 1).

Pour remédier à cette fuite, plusieurs solutions ont été étudiées, et deux ont débouché au stade industriel.

La première consiste à déposer un caoutchouc liquide chargé de résine compensant le manque d'épaisseur localisé (voir fig. 2).

La deuxième consiste en une surépaisseur obtenue directement au moment du moulage du cadre (voir fig. 3).

Dans le cas de l'application laitière de nos électrodialyseurs, c'est cette deuxième solution qui a été retenue, et qui est maintenant largement diffusée chez nos clients. Les résultats obtenus industriellement ont permis d'observer des réductions substantielles de pertes en extrait sec.

\section{b) Utilisation d'électrodes intermédiaires}

La mise au point et l'utilisation d'une ou plusieurs électrodes intermédiaires dans un même empilement nous ont récemment ouvert de nouvelles possibilités :

- augmentation du nombre de cellules par empilement;

- création de plusieurs étages de dessalement dans le même empilement ;

- réduction des tensions continues;

- utilisation de tuyauteries métalliques.

1. Augmentation du nombre de cellules

Pendant longtemps nous avons limité le nombre de cellules* à 350 par empilement. Ce nombre résultait de notre expérience et

* On appelle cellule l'ensemble constitué par :

- une membrane cationique;

- un cadre séparateur ;

- une membrane anionique ;

- un cadre séparateur. 


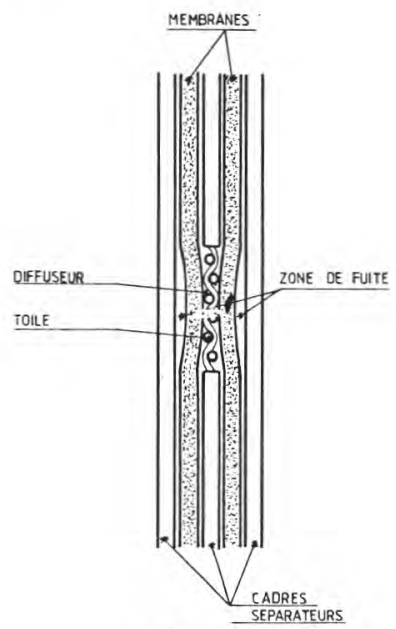

fig. 1

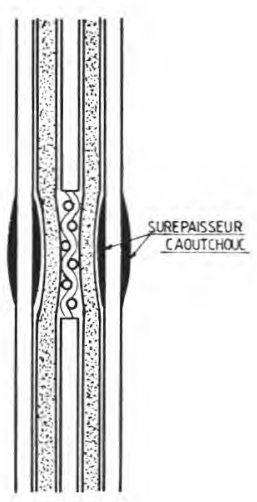

fig. 2

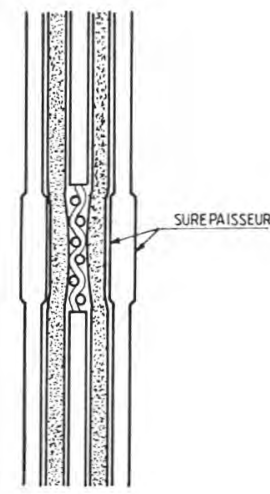

fig. 3

correspondait au maximum de cellules admissibles dans un même système de serrage. (Au-delà, des problèmes de tenue mécanique de l'ensemble apparaissent).

Nous pouvons maintenant réaliser des empilements de 800 cellules en disposant 3 électrodes intermédiaires délimitant 4 «paquets » de 200 cellules. Nous avons ainsi multiplié par près de 2,3 la surface de membranes disponibles dans un même module.

Cela a été rendu possible grâce à la conception de l'Electrode Intermédiaire qui apporte un renfort mécanique indispensable pour un tel nombre de cellules.

$2^{\circ}$ Création de plusieurs étages de dessalement dans un même empilement

On peut imaginer plusieurs variantes possibles et intéressantes obtenues par l'utilisation d'électrodes intermédiaires (voir fig. 4).

Les premiers appareils bénéficiant de ces nouveaux empilements (ou module) ont été destinés au dessalement des eaux saumâtres (Moyen Orient - Afrique du Nord). En effet la salinité des eaux n'excédant généralement pas $5 \mathrm{~g} / 1$ et la résiduelle souhaitée étant voisine de $0,5 \mathrm{~g} / 1$, il est possible de traiter cette eau en passage direct au travers d'un empilement de 4 étages (3 électrodes intermédiaires) si la capacité demandée n'est pas supérieure à $300 \mathrm{~m}^{3} / \mathrm{j}$ (au-delà plusieurs empilements sont nécessaires). 


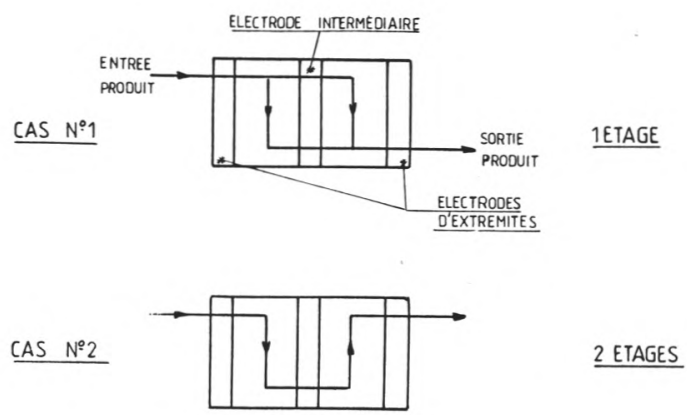

CAS No3

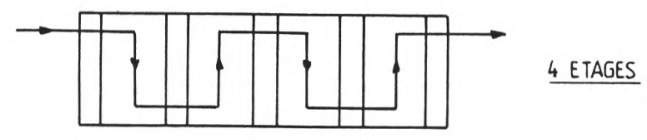

CAS No4

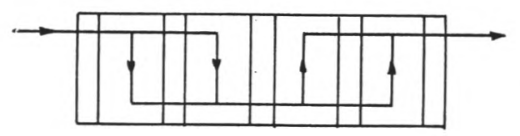

2 ETAGES

CAS NPS

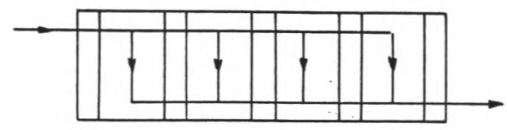

1 ETAGE

fig. 4

30 Diminution de la tension continue

Une retombée intéressante de l'utilisation des électrodes intermédiaires est de réduire les tensions continues.

Exemple : supposons un empilement de 350 cellules alimentées à 2 Volts par cellule.

Sans électrode intermédiaire : $350 \times 2=700 \mathrm{~V}$.

Avec électrode intermédiaire : $\frac{350}{2} \times 2=350 \mathrm{~V}$. 
Le premier cas est défavorable, il conduit entre autre à utiliser du matériel électrique plus coûteux et répondant à une règlementation plus contraignante (au-delà de $600 \mathrm{~V}$ ).

\section{Utilisation de tuyauteries métalliques}

Dans un empilement sans électrode intermédiaire le potentiel appliqué aux électrodes peut être soit flottant, soit fixé ; dans les deux cas, il existe un courant de fuite externe non négligeable qui emprunte les canalisations et qui résulte de la ddp entre les électrodes et les parties métalliques présentes dans les circuits (vannes, pompes, sondes, etc.).

Dans un empilement avec une ou trois électrodes intermédiaires on s'arrange pour que les électrodes d'extrémité (qui reçoivent les canalisations) soient à la masse (voir fig. 5).

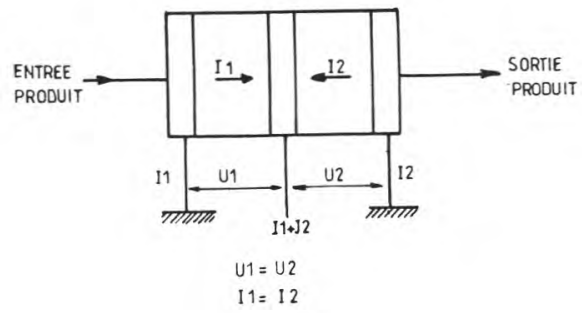

fig. $5 a$

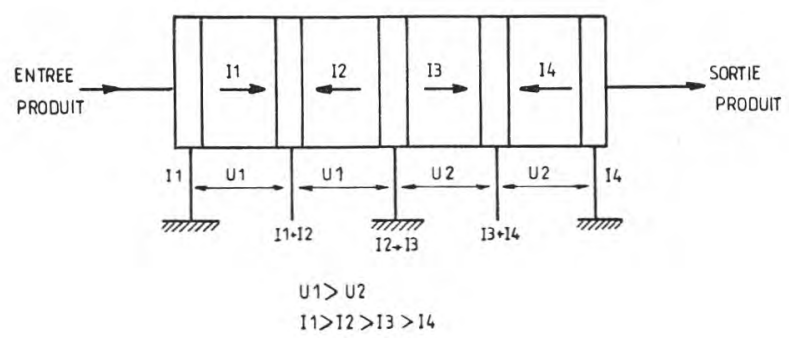

fig. $5 b$

Cette disposition supprime tout risque de fuites externes du courant et permet de minimiser les parties plastiques qui restent évidemment irremplaçables dans l'empilement lui-même et aux abords immédiats.

Sur la base de cette innovation nous avons pu développer une gamme d'appareils tout inox utilisant du matériel typiquement laitier. 
Le développement de ce nouveau type d'installation nous permet d'espérer d'intéressants débouchés dans les pays qui condamnent totalement le PVC et qui suspectent les plastiques en général.

\section{CHOIX DU PROCEDE}

On peut considérer qu'il existe 3 types de procédés pour mettre en œuvre les modules d'électrodialyse :

- le procédé discontinu par cuvée (ou recirculation);

- le procédé continu (ou passage direct) ; Bleed).

- le procédé continu à recirculation partielle (ou Feed and

Ces différents types de procédés offrent des avantages et des inconvénients selon le produit traité, l'extraction demandée et les conditions de traitement. On peut faire appel à l'un ou l'autre suivant le problème posé.

\section{a) Le procédé discontinu (ou recirculation) (fig. 6)}

Dans cette configuration le produit à dessaler tourne en circuit fermé jusqu'à ce que la salinité désirée soit atteinte. Le temps de déminéralisation de la cuvée dépend de son volume, de la minéralisation initiale, du dessalement demandé et de la différence de potentiel appliquée.

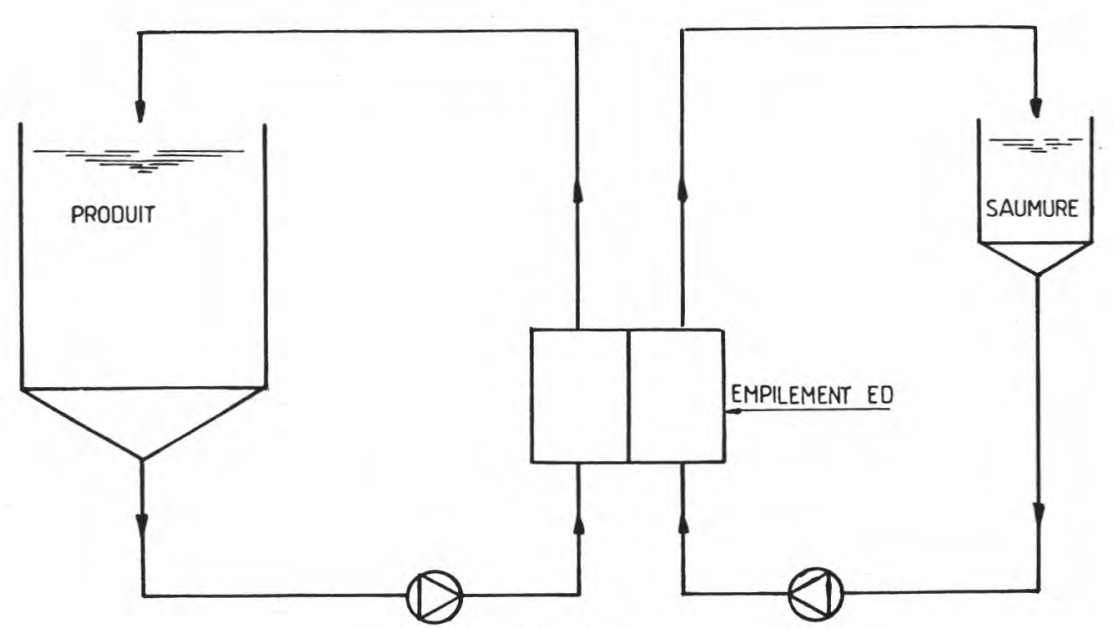

fig. 6 
Dans le cas du lactosérum ce temps ne doit pas dépasser $30 \mathrm{~min}$ pour éviter tout développement bactérien.

Notre Société a utilisé pendant longtemps exclusivement ce procédé pour le traitement de lactosérum. Il présente en effet certains avantages particulièrement intéressants pour ce type de traitement et en particulier :

- une grande souplesse d'utilisation; on peut traiter indifféremment des produits à charges ioniques variables et obtenir différents taux de dessalement sans aménagement particulier en modifiant simplement quelques paramètres de fonctionnement;

- le produit traité est délivré à une qualité constante même si celui-ci varie à l'entrée.

Par contre, le fait même que le procédé soit en discontinu conduit à un environnement plus lourd (stockage - refroidissement réchauffage).

\section{Amélioration du procédé}

La solution la plus simple consiste à appliquer une tension fixe définie par le produit traité et par les limites admissibles par le matériel.

Au cours du cycle de dessalement l'intensité qui en résulte diminue traduisant ainsi l'augmentation de résistivité du produit traité.

Une solution plus élaborée consiste à augmenter progressivement la tension en cours de dessalement tout en prenant garde de rester en deça des conditions limites de fonctionnement.

Sur cette base, nous avons réalisé un Pilote industriel automatique avce tension asservie par thyristors. On fixe alors 2 paramètres : l'intensité maximum de départ, la tension maximum admissible. Ces essais ont montré que l'on pouvait espérer des gains de production de l'ordre de $30 \%$ dans le cas de produit à charge minérale élevée (voir fig. 7).

Industriellement le coût élevé des alimentations électriques thyristorisées ainsi que l'utilisation d'une électronique pointue est pour l'instant, en ce qui nous concerne, un frein au développement de cette technique néanmoins prometteuse.

\section{b) Le procédé en continu (ou passage direct) (fig. 8)}

Dans ce type de procédé le produit ne passe qu'une seule fois dans l'empilement d'électrodialyse. Pour obtenir des taux de dessalement intéressants, il est nécessaire de disposer plusieurs étages de dessalement, soit intégrés dans le même empilement (EI), soit avec plusieurs empilements. 


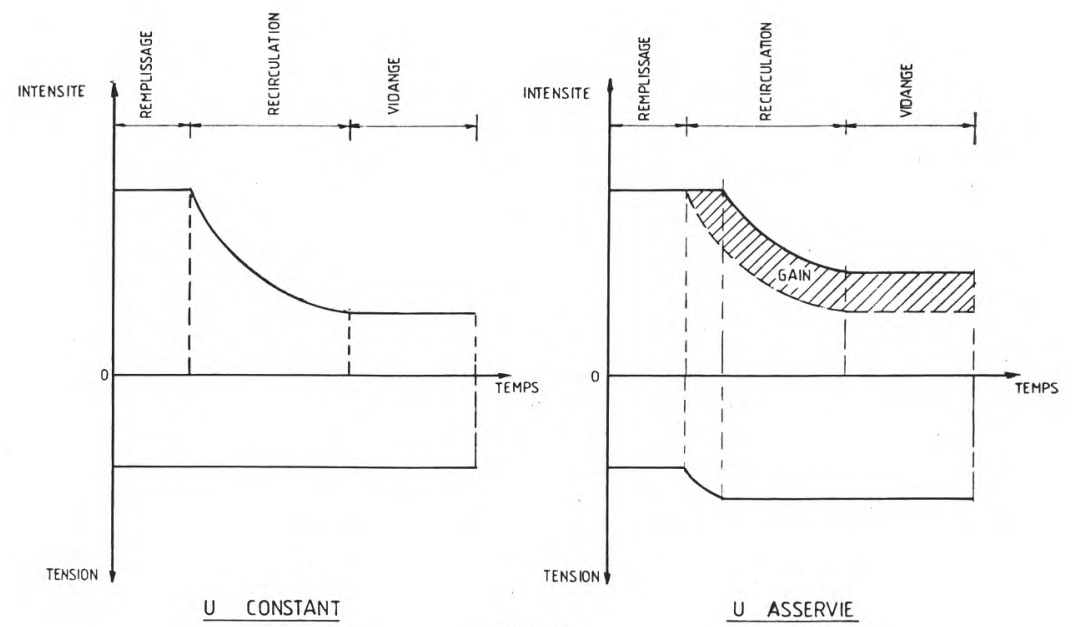

fig. 7
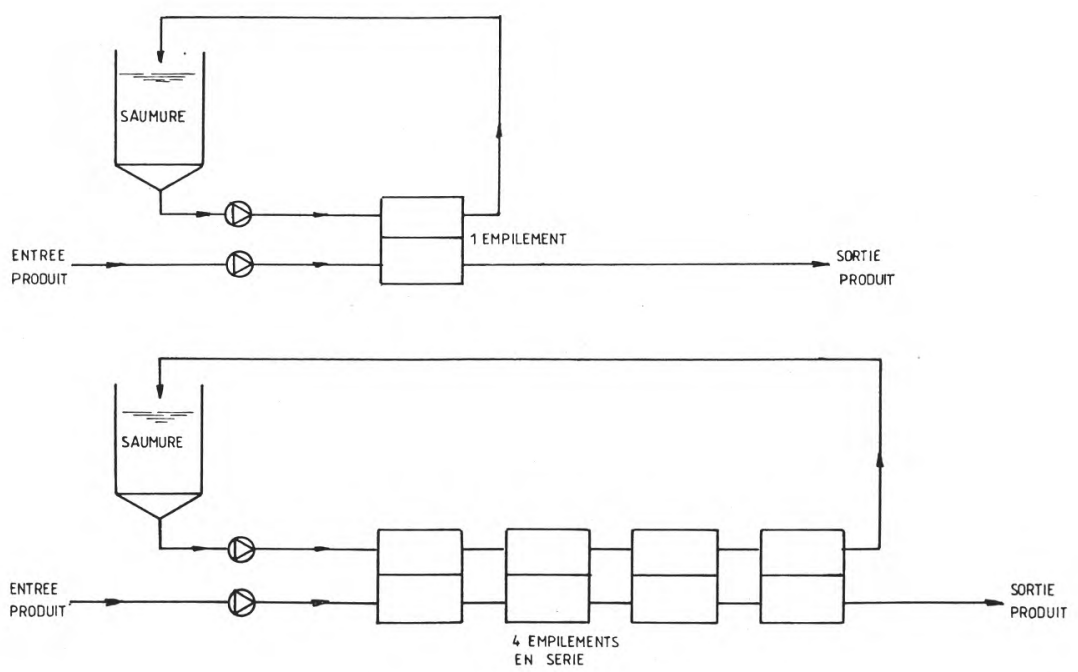

fig. 8

Dans le cas des lactosérums le type de procédé reste d'un emploi très limité : le nombre d'étages restant faible (inférieur à 4) en raison des pertes de charges, on ne peut pas prétendre à des taux de dessalement élevés.

Exemple : du sérum $6 \%$ ES traité en 4 étages conduit à environ $50 \%$ de déminéralisation. 
C'est dans notre activité en dessalement des eaux saumâtres que ce procédé connaît le plus grand développement (voir § II-a).

\section{c) Procédé continu à recirculation (fig. 9)}

Ce procédé consiste à renvoyer en tête du module une fraction plus ou moins importante de la production. Il présente un intérêt certain dans le traitement des lactosérums car il offre plus de possibilités que le passage direct tout en conservant la fonction production en continu. On a alors la possibilité d'intégrer une installation de ce type dans une ligne de traitement avec un minimum de stockage et en minimisant les refroidissements et les réchauffages.

Ce procédé est d'autant plus intéressant et performant que la teneur en sels du produit à traiter est importante et que la résiduelle demandée reste élevée. On traitera essentiellement des lactosérums préconcentrés entre 20 et $24 \%$.

Nous avons récemment fabriqué et installé plusieurs installations de ce type.

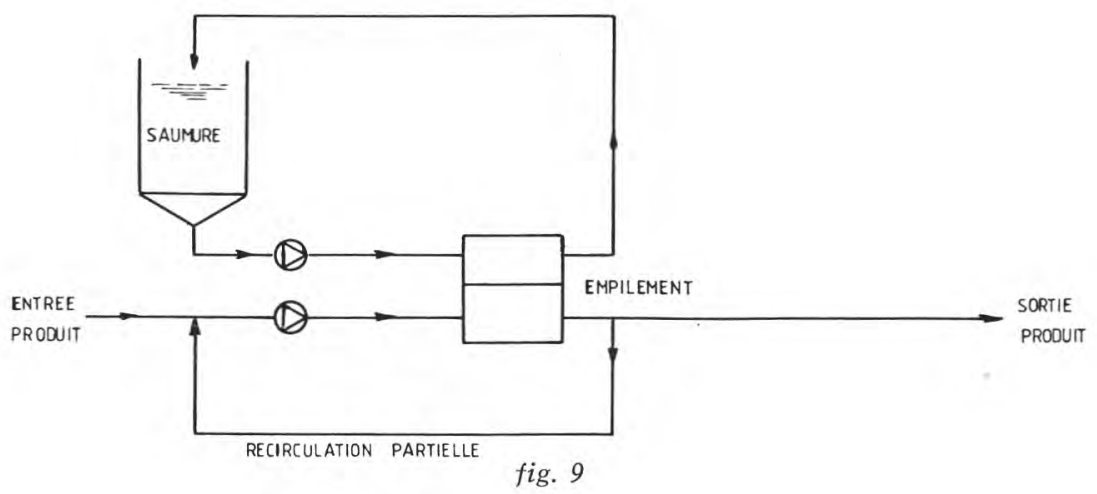

\section{CONCLUSION}

Les progrès du procédé d'électrodialyse dans les industries laitières depuis 10 ans ont résulté davantage de perfectionnements technologiques sur les appareils que d'innovations fondamentales. Certains d'entre eux, mis au point avec une société spécialisée d'un détergent adapté au nettoyage des électrodialyseurs, dispositifs de lavage automatique (CIP), n'ont pu trouver leur place dans ce bref exposé. 